\title{
A distributed message in message aware concurrent transmission protocol in IEEE 802.11 WLANs
}

\author{
Young-myoung Kang ${ }^{1}$, Joon Yoo ${ }^{2^{*}}$, Joonsoo Lee $^{3}$ and Chong-kwon Kim ${ }^{1}$
}

\begin{abstract}
The IEEE 802.11 distributed coordination function (DCF) employs the carrier sense technology to avoid frame collisions. However, recent measurement studies demonstrate that the physical layer (PHY) capture effect frequently occurs; even when frames collide, one of them can be decoded successfully if its relative signal strength is high enough. Furthermore, a new wireless PHY technology, called Message In Message (MIM), adopts an advanced preamble detection function to enhance the PHY capture effect. To fully exploit MIM in multi-collision environments, frame transmission orders have to be carefully scheduled. It also requires tight time synchronization at multiple access points (APs), thus induces large overheads. In this article, we propose an opportunistic concurrent transmission protocol called Distributed Opportunistic MIM-aware Concurrent Transmission (DOMCT) which exploits the MIM functionality in a distributed manner obliterating the centralized control. In DOMCT, APs first prepare interference MAPs to discover the possible simultaneous MIM transmission opportunities. Detecting the inadvertent frame transmission from a neighboring AP, an AP transmits another frame intentionally if both frames can successfully be decoded at destination nodes by the MIM capture effect. Through both analysis and extensive ns-2 simulations, we show that DOMCT outperforms the legacy DCF by up to $61 \%$ and observe comparable performance to that of the centralized approach.
\end{abstract}

Keyword: WLANs, Concurrent transmission, Message in message (MIM), Capture effect, Scheduling

\section{Introduction}

To support the ever growing demands for mobile communications, IEEE 802.11 wireless LANs (WLAN) have continuously evolved to higher speed variants $[1,2]$. Despite the improvements in physical layer (PHY) technologies, the goodput of WLANs does not increase in linear proportion to the PHY speed [3]. One limiting factor is large MAC overheads such as back-off time, long protocol header, ACK, and various inter-frame shifts. Many clever schemes that reduce the MAC overheads have been proposed including frame aggregation [1] and binary back-off optimization $[4,5]$. These schemes are called as the temporal approach because they reduce the time required for MAC layer operations.

\footnotetext{
* Correspondence: joon.yoo@gachon.ac.kr

${ }^{2}$ Department of Software Design and Management, Gachon University, Seongnam, Korea

Full list of author information is available at the end of the article
}

This article deals with the interference, another factor that degrades the WLANs performance. Interference is considered as one of the most important factors that decides wireless network throughput per unit area. Interference avoidance or reduction in multi-hop wireless networks has been the subject of active research during last several years and a plethora of mechanisms [6-8] that mitigate the effect of interference have been introduced. These mechanisms are referred to as a spatial approach because they essentially try to increase the number of simultaneous transmissions per unit area.

Advanced signal processing makes it possible to decode one of simultaneously received frames-i.e., collided frames-successfully if certain conditions are satisfied. The conventional wisdom is that if two or more frames arrive at a receiver at the same time then all of them fail and result in a collision. Recent observations confirm the PHY capture effect [9-11]; if two frames collide within a preamble period, a receiver can successfully lock on to a 
stronger signal if it is sufficiently more powerful than the other signal. The PHY capture effect improves the system goodput in a single collision domain. However, its performance gain in a multi-hop network is limited because collisions due to hidden terminals can occur randomly and the probability of preamble collisions is not great.

Contrary to the PHY capture effect which is rather an coincidental outcome, Message in Message (MIM) [10-13] is a result of ingenious engineering effort. Modern MIMcapable NICs, such as Atheros [14], can capture the intended signal with higher SINR $(\geq 10 \mathrm{~dB})$ even when the intended signal arrives after the preamble of an interference signal [10]. The rationale behind the different capture behaviors is that MIM-capable NICs continuously search for a new preamble even if it has already locked on to a preceded frame while MIM-incapable NICs do not search for a new preamble once it synchronizes and locks on to the frame. Figure 1 explains the difference between the $\mathrm{PHY}$ and MIM captures.

MIM may not be very useful in a single collision domain because the carrier sense function prevents the frame transmission during busy periods. However, in multiple collision domains, MIM enables successful deliveries of otherwise interfering signals. Santhapuri et al. [12,15] proposed an MIM-aware centralized packet scheduling algorithm called Shuffle that supports concurrent transmissions from multiple APs. Suppose there are two signals interfering with each other. The basic rule of Shuffle is to transmit a relatively weaker signal before a relatively stronger signal so that both signals are successfully decoded via the MIM capture. Shuffle employs a centralized controller that coordinates frame transmission orders of all the APs in consideration. Shuffle, the first approach that deliberately exploits the MIM capability, suffers from the usual drawbacks inherent to the centralized approaches. In addition, it can only be applied to a single autonomous system and also requires tight time synchronization among the APs.

In this article, we propose a Distributed Opportunistic MIM-aware Concurrent Transmission (DOMCT) protocol. In DOMCT, backlogged APs continuously overhear the transmission of neighboring AP, looking for the opportunities of concurrent transmission via MIM. Observing such opportunities, APs autonomously trigger impromptu concurrent transmissions. In a basic mode, APs initiate frame transmission in a random order. DOMCT achieves additional performance gains by employing enhanced mechanisms such as a per-station queue strategy and transmission ranking (TR). By using the per-station queue strategy, DOMCT effectively fetches the MIM capable frames in real time. On the other hand, TR boosts the throughput performance by increasing the opportunities of concurrent transmissions.

Our main contributions are summarized as follows:

- We propose a DOMCT protocol. Since DOMCT operates in a distributed manner, it eliminates the tight time synchronization requirement and high control overhead of the centralized scheme. Furthermore, the distributed nature of DOMCT supports backward compatibility with the legacy IEEE 802.11 Distributed Coordination Function (DCF).

- We analyze the probability of the MIM capture in a two APs scenario to verify the potential gain from the MIM-aware concurrent transmissions. The result shows that MIM capture can significantly improve the channel utilization.

- We devise a per-station queue strategy and TR so that DOMCT achieves additional performance gains by employing those enhanced mechanisms.

\begin{tabular}{|cc|}
\hline$\vdots$ & \\
\hline preamble & Interference \\
\hline$\vdots$ & \\
\hline preamble & Intended Signal \\
\hline
\end{tabular}

PHY Capture : Success at high SINR ( $>=10 \mathrm{~dB})$. An intended signal should arrive within the preamble period of an interference signal.

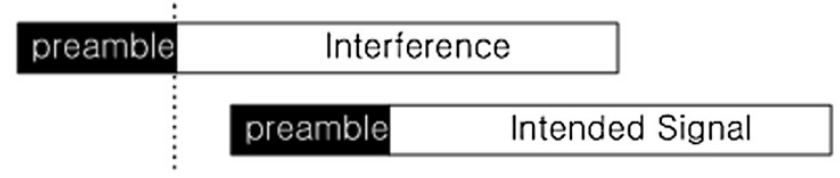

MIM : Success at high SINR ( $>=10 \mathrm{~dB}$ ). An intended signal can be captured even though it arrives after the preamble period of an Interference signal.

Figure 1 PHY capture versus MIM capture. 
- We compare the system throughput of DOMCT, DCF, and Shuffle. Our ns-2 simulation shows that DOMCT improves the system throughput by $61 \%$ on average compared to the legacy DCF. Furthermore, DOMCT achieves the performance comparable to Shuffle, the state-of-the-art centralized approach.

The rest of the article is organized as follows. We present the related work in the next section. The detailed description of DOMCT protocol including its enhancements and the analysis of the MIM opportunity are given in "DOMCT protocol" section. "Simulation results" section shows the simulation results. Finally, we conclude the article and provide our future work in "Conclusion" section.

\section{Related work}

Various previous studies on the PHY capture effect [9,16-19] mainly focused on increasing the PHY capture probability. Basically, the PHY capture effect is a coincidental outcome where only one of the collided frames may survive. In contrast, MIM has the capability to capture multiple frames from the collided frames. The authors of $[10,11]$ thoroughly carried out empirical experiments and quantify the threshold that enables MIM captures. Shuffle [12,15] implements MAC layer frame scheduling in order to increase MIM concurrent transmissions. However, Shuffle aggressively disables carrier sensing and carries out consecutive concurrent transmissions causing legacy DCF devices to starve. In contrast, DOMCT increases the system throughput via opportunistic concurrent transmissions thus protecting ongoing transmissions of other APs.

A plethora of advanced signal processing mechanisms aim at reducing or eliminating potential interferences [20-22] has been proposed. SIC [20] decodes a relatively stronger signal from overlapped signals and then distracts a weaker signal by subtracting the stronger signal from the overlapped signals. This mechanism requires complex symbol level signal manipulation. Similarly, ZigZag [23] requires signal manipulation to recover the signal from the collided frames. It does not increase the wireless capacity but only reduces the number of retransmissions similar to PPR [24]. On the other hand, IAC [21], SAM [22], CSMA/CN [25] support concurrent transmissions by the interference alignment and interference cancellation using multiple signal streams obtainable in MIMO environments. It is worthwhile noting that our proposal, DOMCT, targets single antenna systems.

Some of the centralized architectures [15,26-30] provide the concurrent transmissions through MAC frame scheduling. However, these mechanisms do not take the MIM functionality into account except for Shuffle [15]. Therefore, they miss the potential concurrency gains from the MIM opportunities. CMAP [31] and OCP [32] support concurrent transmission in a distributed manner. CMAP [31] constructs a conflict map via empirical evaluations and permits concurrent transmissions from exposed terminals. This method is similar to our proposed scheme in the sense that it selectively activates carrier sensing and permits an additional concurrent transmission if it does not corrupt the ongoing frame. However, CMAP makes concurrent transmission decisions based on the historical concurrent transmission results (i.e., success/failure) instead of the explicit SINR-based measurement. OCP [32] also constructs an interference map (IMAP) and conducts concurrent transmission opportunistically. However, as in CMAP, OCP decides the feasibility of concurrent transmissions based on empirical evaluations (i.e., success/failure). In addition, OCP requires changes in the frame structure since it adds a post-amble at the end of the frame.

Assessing the exact interference between contending links is crucial to the system performance because the results of concurrent transmission is tightly coupled with the interference relationship. The authors of $[27,28]$ introduced an SINR-based conflict map. These schemes are only applicable in a static environment due to large measurement overheads. In contrast, our solution can be operated both in static and mobile environments since we adopt a lightweight online estimation scheme that is similar to micro-probing [33] in constructing the IMAP.

\section{DOMCT protocol DOMCT}

DOMCT consists of two stages [34]. First, an IMAP is constructed by APs to find out the interference relations between the nodes. Then, based on the IMAP, frames are concurrently transmitted when the MIM capture threshold requirements are satisfied.

\section{IMAP}

Each AP constructs an IMAP [15] using a method similar to micro-probing [33]. Figure 2 describes the IMAP construction algorithm. Each AP builds its own IMAP by employing the following operations. At the initial phase, each AP gathers the channel quality information from its clients. Each client piggybacks the SINR value from the AP to itself in ACK frames to APs. When an AP overhears an inadvertent frame transmission of neighboring AP (line 9), it decodes the MAC header and identifies the link ID (APstation pair) of the overheard frame (line 10). Then, it initiates a concurrent transmission to its client immediately after the MAC header part of the ongoing frame (line 12). After this intentional concurrent transmission attempt, the AP looks up the SINR value piggybacked in the ACK frame and records it in the IMAP. Each AP shares its IMAP with 


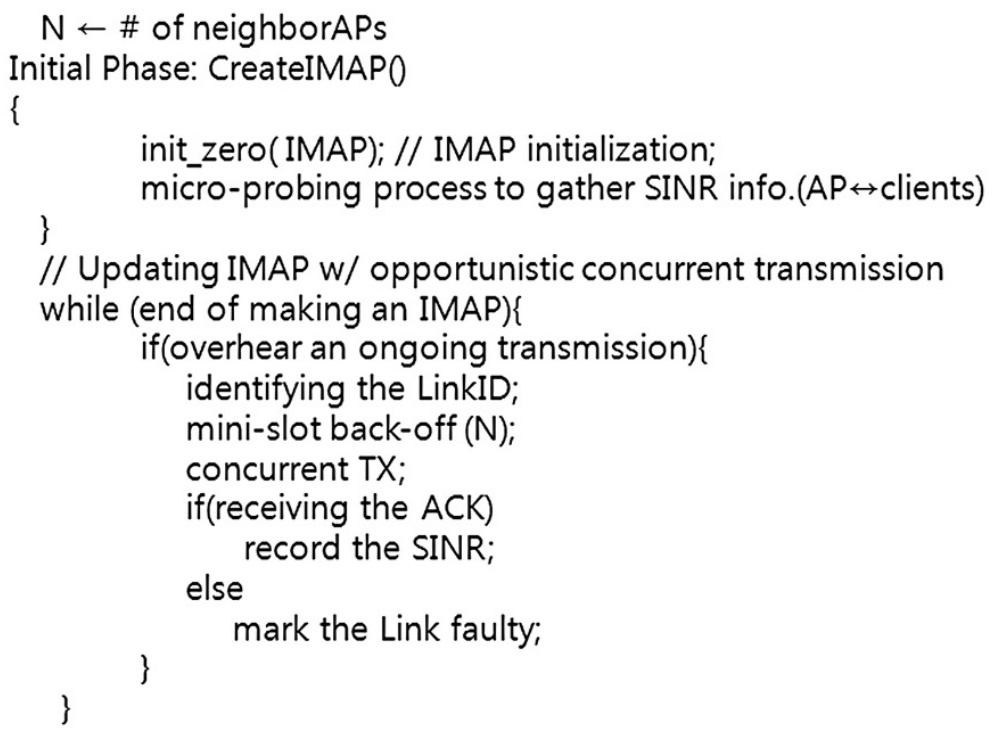

Figure 2 IMAP construction algorithm.

its neighboring APs through beacon exchanges. This operation is repeated for all other links until the entire IMAP is finally established.

It is possible that more than one AP may try this operation resulting in collisions or ACK losses. To minimize the collisions among opportunistic APs, each AP estimates the number of neighbor APs [35] and takes a mini-slot back-off [36] at the end of MAC header part of the ongoing frame (line 11). The back-off value is set in proportion to the number of contending APs. We set the range of the mini-slot back-off window as same as the binary exponential back-off window counter. For example, if there are two APs (AP1 and AP2) contending for concurrent transmissions, each AP determines minislot back-off counter based on the number of contending APs (e.g., AP1: 1, AP2: 3). AP1 conducts carrier sensing at the first mini-slot and finds it as idle. Thus, AP1 initiates concurrent transmission. AP2 tries to access the medium at the third mini-slot; however, it finds the preamble transmission of AP2, and gives up concurrent transmission. If an ACK frame is lost, we imply it as a concurrent transmission failure. Hence, the AP marks these links as a failed link in the IMAP. Thereafter, it avoids using them in order to protect the transmission of the neighboring APs (line 13-16).

When clients disassociated from the current APs, then IMAP should be updated accordingly. The appropriate IMAP update interval depends on several factors. Shorter update intervals waste the wireless bandwidth due to the control overhead in an unsaturated condition. On the other hand, longer update intervals fail to cope with dynamic channel fluctuation and user mobility. Therefore, instead of using a fixed update period, the IMAP is updated both periodically and opportunistically as follows

$$
\text { PeriodicUpdateInterval }=\delta * \text { Periodic }
$$

where $\delta$ is controlled in proportion to the arrival rate of the traffic volume. The periodic updates (e.g., 1 s) refresh the entire contents in the IMAP. In our simulations, the average update period was measured as $0.64 \mathrm{~ms}$. In addition to the periodic updates, each time an AP conducts a concurrent transmission, the result-e.g., success or failure-is opportunistically updated in the IMAP. Therefore, the IMAP is kept up-to-date and it also copes with timevarying channel and user mobility.

\section{Opportunistic concurrent transmission}

The basic operation of the opportunistic concurrent transmission algorithm is described in Figure 3 . When an AP overhears transmission from a neighbor AP, it looks up the IMAP to check whether a concurrent transmission is feasible or not (lines 1-3). The concurrent transmission is admitted if the following conditions are met. (1) The SINR of the ongoing frame is not less than the first frame capture threshold $\left(\theta^{F} \geq 4 \mathrm{~dB}\right.$ in 802.11a radio) and the SINR of the concurrent frame is greater than or equal to the last frame capture threshold $\left(\theta^{L} \geq 10 \mathrm{~dB}\right.$ in 802.11a radio). (2) The SINRs of both frames satisfy the predetermined RX sensitivity (ex. $-88 \mathrm{dBm}$ for $6 \mathrm{Mbps}$ by the 802.11 a standard) (lines 4 and 11-16). To minimize collisions, it uses a minislot back-off at the end of each MAC header of the ongoing frame (line 5).

DOMCT requires a more sophisticated ACK processing mechanism in the unicast transmission. Even though two frames are delivered successfully, their ACKs 


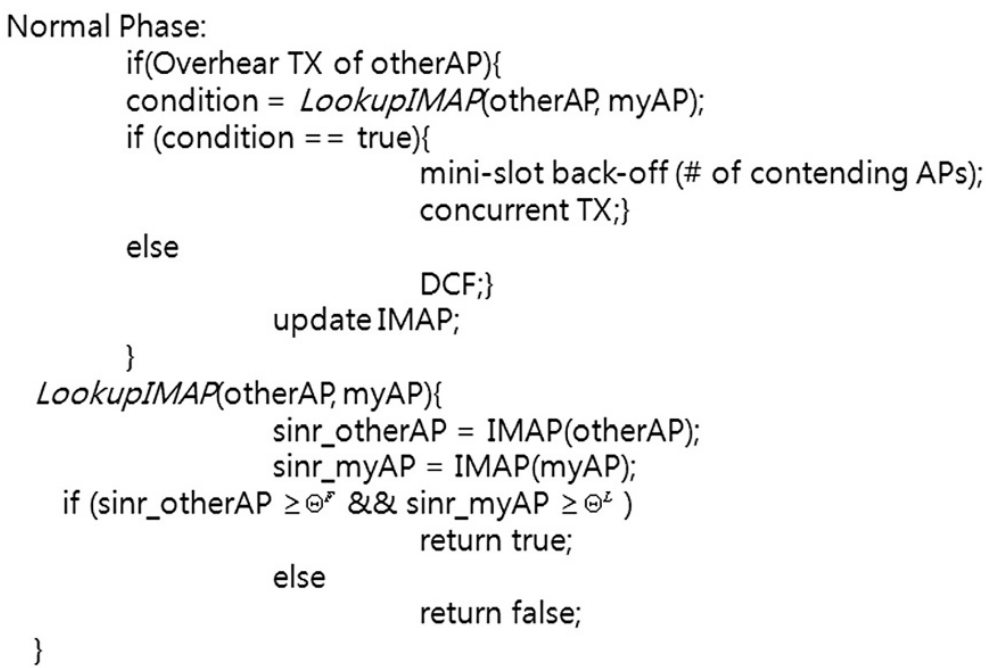

Figure 3 Opportunistic concurrent transmission algorithm.

can be collided at APs. We avoid ACK collisions by serialized scheduling ACK frames. For example, AP2 knows the ACK transmission time of AP1 by using the MAC header information of AP1's frame. AP2 avoids overlapping of its own ACK and AP1's ACK by delaying its ACK transmission until the end of ACK transmission to AP1. Note that our simulation results demonstrate that DOMCT operates well even without ACK serializing, since ACKs are typically transmitted at the basic data rate and the frame length is relatively short. Another possible solution is the piggybacking of SINR reports in the data frames to reduce the adverse effects of ACK collision. Besides ACK collisions, data and ACK frames can collide also. We can avoid this problem making the concurrent transmission completes at the same time the first data transmission finishes.

Let us explain the DOMCT operation with an example shown in Figure 4. There are two APs (AP1, AP2) and three clients (R1, R2, and R3). Solid arrows represent a link between AP and client and dashed lines indicate potential interference relationships between concurrent transmissions. Both APs are located within the transmission range

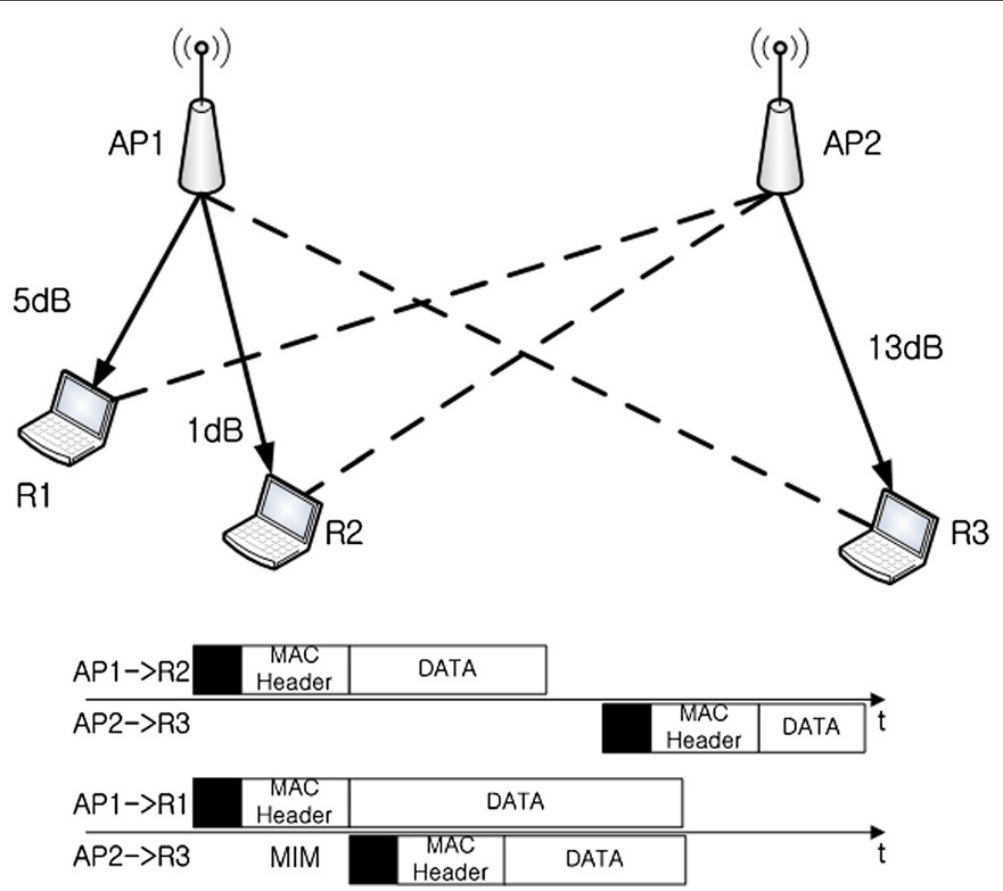

Figure 4 The operation of DOMCT protocol. 
of each other as DOMCT requires the APs to overhear and examine the MAC header from the neighboring APs in order to identify the link ID. This implies that DOMCT is well suited for densely deployed WLANs which are common cases nowadays. The figure also shows the received SINR when frames are transmitted concurrently. We assume that both APs operate on the same frequency channel.

In this example, we can identify two cases of concurrent transmissions: (i) AP1 $\rightarrow \mathrm{R} 1$ and $\mathrm{AP} 2 \rightarrow \mathrm{R} 3$ transmissions, and (ii) AP1 $\rightarrow$ R2 and AP2 $\rightarrow$ R3. In the first case where AP1 and AP2 are transmitting frames to their corresponding clients R1 and R3 concurrently, both transmissions are successful. As long as AP1 $\rightarrow$ R1 transmission precedes $\mathrm{AP} 2 \rightarrow \mathrm{R} 3$ transmission, a concurrent transmission of AP2 does not corrupt AP1's packet because both frames satisfy the MIM capture threshold requirements (i.e., preceded packet $\geq 4 \mathrm{~dB}$, followed packet $\geq 10 \mathrm{~dB}$ ). On the other hand, in the second case, AP1's transmission may result in a collision while AP2's transmission is successful. That is because the SINR at R3 (13 dB) satisfies the MIM capture threshold while the SINR at R2 (1 dB) does not. In this case, AP2 should not transmit in order to protect the transmission from AP1.

Figure 5 explains the different transmission scenarios of DOMCT depending on the following three cases of MIM opportunity. First, if both AP1 and AP2 satisfy the MIM capture threshold requirements $(A \geq 10 \mathrm{~dB}, B \geq 10 \mathrm{~dB})$, then concurrent transmissions are allowed regardless of transmission order. Second, if both the APs do not satisfy the MIM capture condition, they should obey the legacy DCF of single transmission at any time. Finally, if the results of concurrent transmissions depend on the transmission order, regulating the transmission order is important. In Figure 5, concurrent transmission is possible when the transmission of AP2 follows the transmission of AP1, whereas the reversed transmission order makes the MIM capture unfeasible. In this case, AP2 may intentionally delay its transmissions in order to increase the opportunity of MIM capture.

\section{Increasing MIM opportunities}

In DOMCT, it is important to provide the concurrent transmission opportunities as many as possible to increase the system throughput. We propose a per-station queue strategy and a TR mechanism to supplement the basic operation of DOMCT.

\section{Per-station queue strategy}

Even when an AP is given concurrent transmission opportunities, it may lose the chances due to the inappropriate frame sequence in its queue. We explain this situation using an example shown in Figure 6a. When AP2 overhears the transmission on the link $1(\mathrm{AP} 1 \rightarrow \mathrm{R} 1)$, AP2 can concurrently transmit to link 3 (AP2 $\rightarrow$ R3). However, the frame for the link $2(\mathrm{AP} 2 \rightarrow \mathrm{R} 2)$ at the head of queue blocks the concurrent transmission opportunity.

Let each AP use the per-station queue. If an AP has at least one frame for each client and the MIM capture condition is satisfied then, the per-station queue strategy always enables the concurrent transmission by fetching the appropriate frame from the per-station queue onthe-fly. In Figure 6b, when AP2 overhears the transmission on the link 1 (AP1 $\rightarrow$ R1), AP2 can fetch a frame to R3. Now, two frames (AP1 $\rightarrow$ R1, AP2 $\rightarrow$ R3) can be transmitted concurrently via MIM. Thus, the per-station

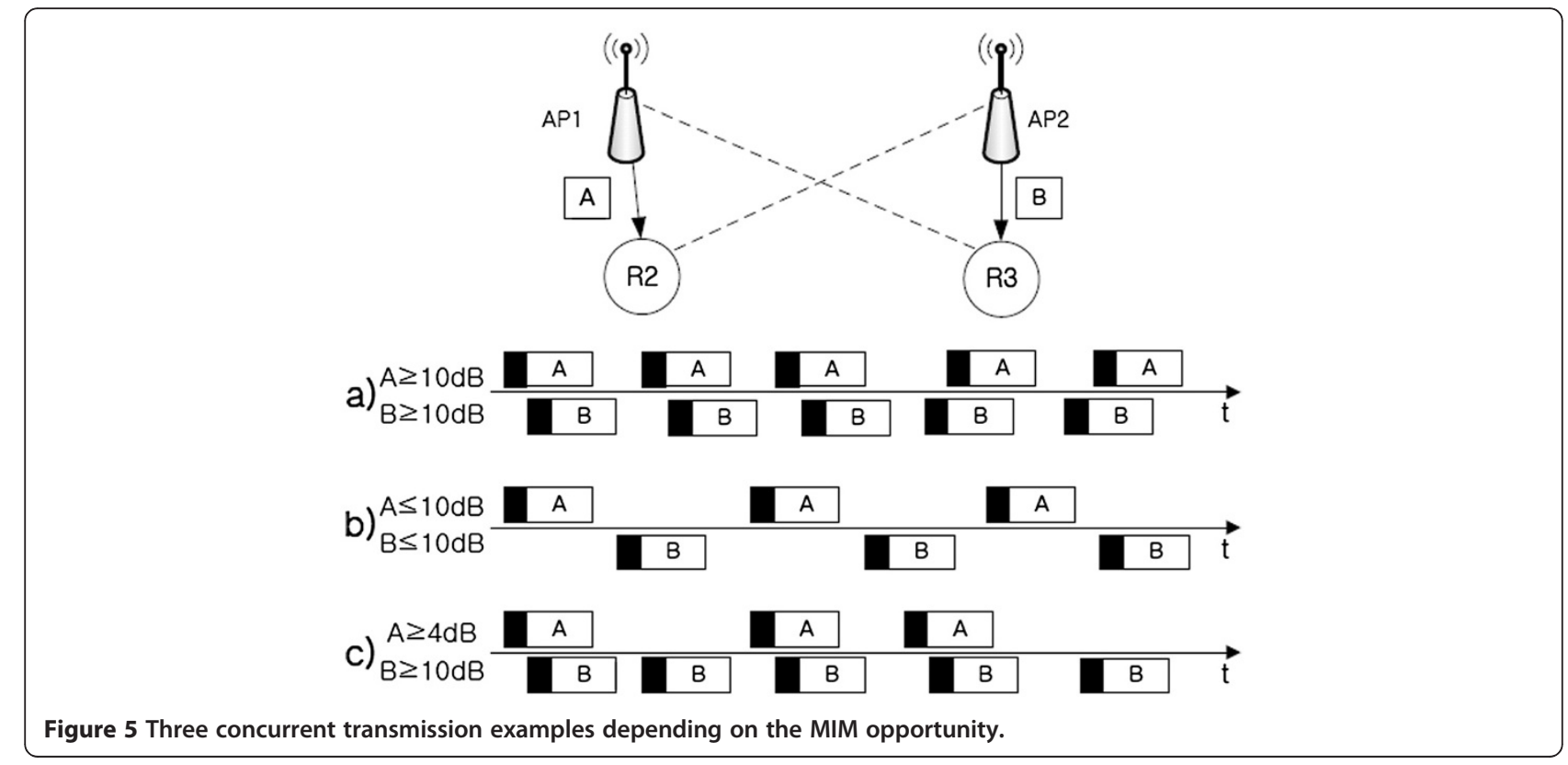




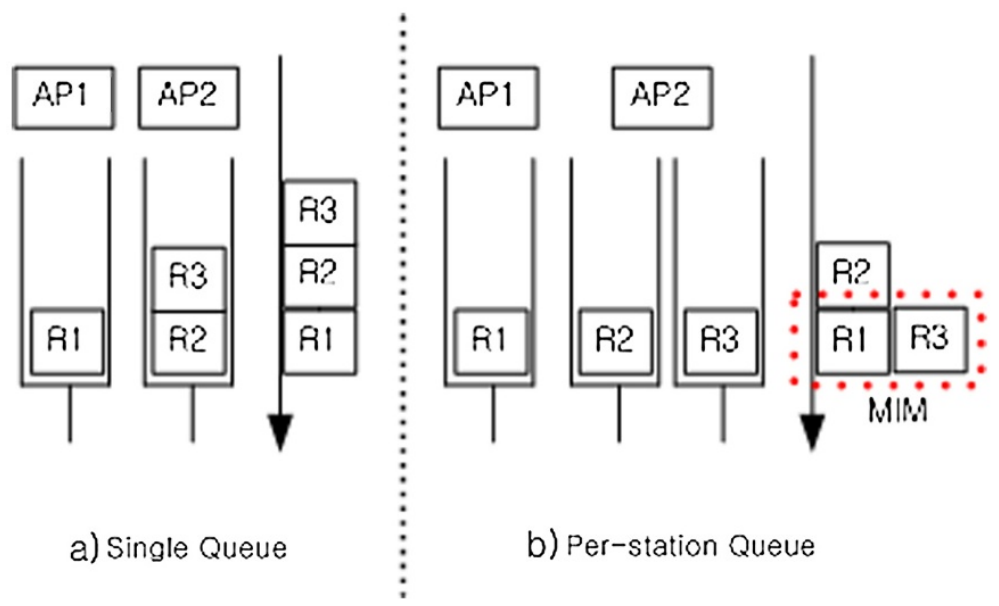

Figure 6 Per-station queue strategy.

queue eliminates the blocking problem and increases the concurrent transmission opportunity.

The per-station queue strategy can be regulated depending on the performance criteria such as throughput, delay, or fairness. To increase the throughput, the AP fetches the packet that belongs to the concurrent transmission links from the per-station queue. For a minimum delay, the AP fetches the packet in a FIFO manner. If fairness is the main objective, then the max-min fairness or proportional fairness may be employed. In this article, we choose throughput as an objective metric.

\section{TR}

We devise TR to increase the number of MIM-enabled concurrent links. In the TR, the AP gives priority to each
AP-client link based on the potential number of concurrent transmission opportunities. We explain the basic idea of TR with an example shown in Figure 7. Each AP has a ranking table whose entry represents the ranking of a link to each client. An entry consists of two fields, $F$ and $L$. The $F$ field is the number of neighboring links that can be activated concurrently with the current link. In other words, the neighboring links in the $F$ field can transmit using MIM capture, by following the frame transmission of the current link. Meanwhile, the $L$ filed shows the number of neighboring links that are compatible as preceding transmissions to the current link. For instance, if AP2 transmits a frame earlier than other APs, three links (i.e., $\mathrm{AP}_{1} \rightarrow C_{11}$, $\mathrm{AP}_{4} \rightarrow C_{41}$ and $\mathrm{AP}_{3} \rightarrow C_{31}$ ) satisfy the MIM capture conditions, thus $F=3$. Meanwhile, delaying the transmission of

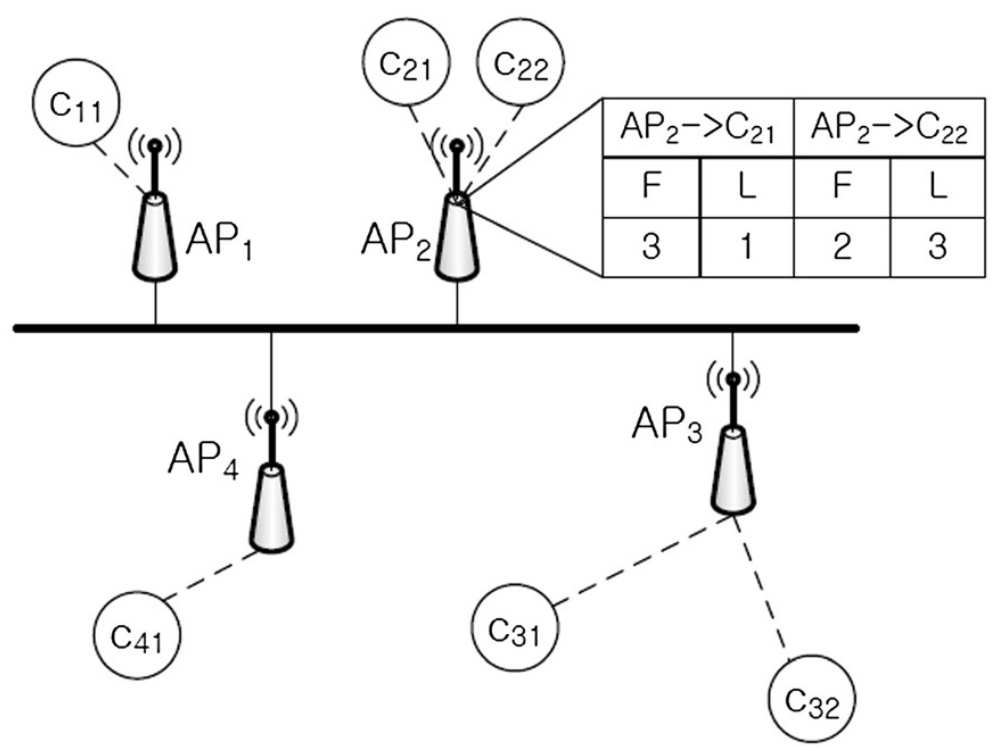

Figure 7 Enhancing the number of concurrent transmission links via TR. 
$\mathrm{AP}_{2}$ after the transmission of other APs enables only one concurrent link (i.e., $\mathrm{AP}_{3} \rightarrow C_{32}$ ), thus $L=1$. Now, $\mathrm{AP}_{i}$ determines its transmission order by computing the $F / L$ of each AP-client link. If $F / L$ is greater than $1, \mathrm{AP}_{i}$ transmits frames immediately. Otherwise it defers to increase the probability of concurrent transmissions.

It is necessary to acquire the inter frame transmission time of other APs in order to employ the TR. Thus, the TR can be activated with the persistent traffic such as VoIP and video on-demand $(\mathrm{VoD})$ because the packet generation intervals of these applications are constant or predictable.

\section{MIM opportunity analysis}

To demonstrate the potential improvement of MIMaware opportunistic concurrent transmission over legacy DCF, we analyzed the MIM capture probability in a scenario where there are two contending links $(\mathrm{AP} 1 \rightarrow \mathrm{R} 1$ and AP2 $\rightarrow \mathrm{R} 2$ ), as shown in Figure 8. Without the loss of generality, we assume that AP1 always precedes AP2 in transmission. We ignore background noise for simplicity. Let $p i$ be the transmission power of AP $i$ ( $i \in$ set of AP) and $G_{i j}$ be the channel gain between AP $i$ and client $j$ ( $j \in$ set of client). AP $i$ is the transmitter, and the APs other than the transmitter AP $i$ are all potential interferers. Then, the SIR (signal-to-interference ratio) at client $j$ is expressed as follows

$$
\operatorname{SIR}_{i j}=\frac{p_{i} G_{i j}}{\sum_{k \neq i, j} p_{k} G_{k j}}
$$

where

$$
G_{i j}=d_{i j}^{\phi}
$$

In (1), $d_{i} j^{(-\phi)}$ denotes the distance between AP $i$ and client $j$ and $\phi$ is the pathloss exponent. We set $\phi$ to four assuming an indoor environment. Suppose that the transmission powers of all APs are homogeneous and no fading occurs (i.e., Free-space model), the SIR is determined only by the distance between the transmitter and the receiver. Therefore, the $\mathrm{SIR}$ (in $\mathrm{dB}$ ) at $\mathrm{R} 1$ is given by

$$
\operatorname{SIR}_{\mathrm{AP} 1 \mathrm{R} 1}=10 \log \left(\frac{d_{A P 2 \rightarrow R 1}}{d_{A P 1 \rightarrow R 1}}\right)^{\phi}
$$

The SIR of R2 is derived in a similar way. For more detailed analysis, refer to our previous work [37]. Next, we compute the probability of the MIM capture by applying the following two conditions.

$$
\begin{aligned}
& S I R_{\mathrm{RI}} \geq 4 \mathrm{~dB} \text { (First MIM capture) } \\
& S I R_{R 2} \geq 10 \mathrm{~dB} \text { (Second MIM capture) }
\end{aligned}
$$

For ease of understanding, we explain the results with Figure 8 . The regions marked (a) and (b) in Figure 8 represent the first and second MIM capture conditions (i.e., Equations (3) and (4), respectively), respectively. We set the transmission range of an AP as $250 \mathrm{~m}$. By solving (5) below, we finally have the probabilities of the MIM capture as a function of distance between the APs as shown in Figure 9.

$$
\left(\frac{\text { (a) }}{\mathrm{TX}_{\text {Range(AP1) }}} \times \frac{\text { (b) }}{\mathrm{TX}_{\text {Range }(\mathrm{AP} 2)}}\right)
$$

We observe that the MIM capture occurs frequently as the distance between two APs increases. Moreover, if we consider the case of the reversed transmission order where AP2 initiates a transmission first, the total MIM capture probability will increase substantially. This results show that the MIM capture has a large potential to improve spatial reuse in the current wireless networks, where APs are densely deployed.

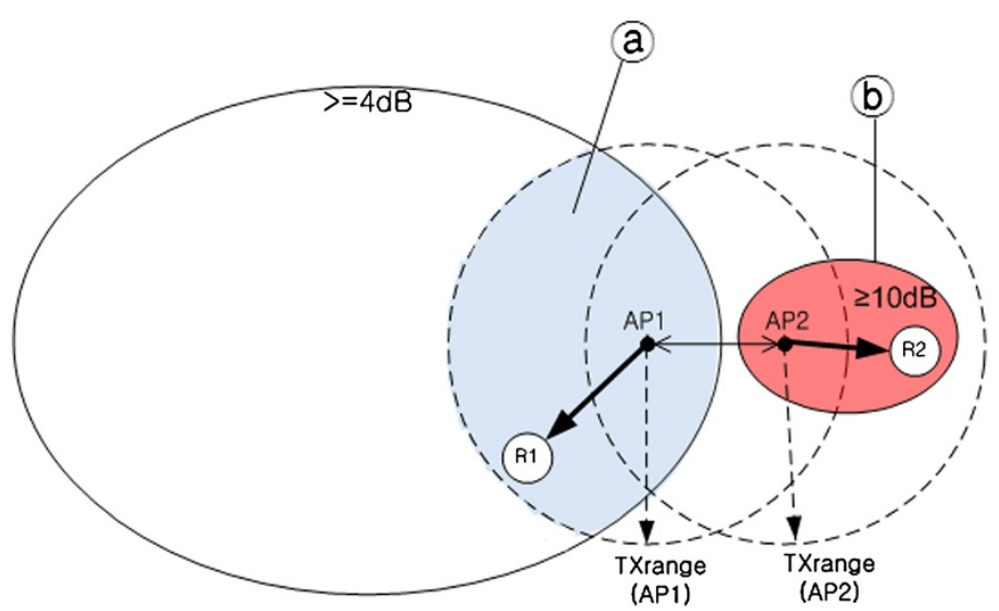

Figure $8 \mathrm{~A}$ two contending links topology used in the analysis. 


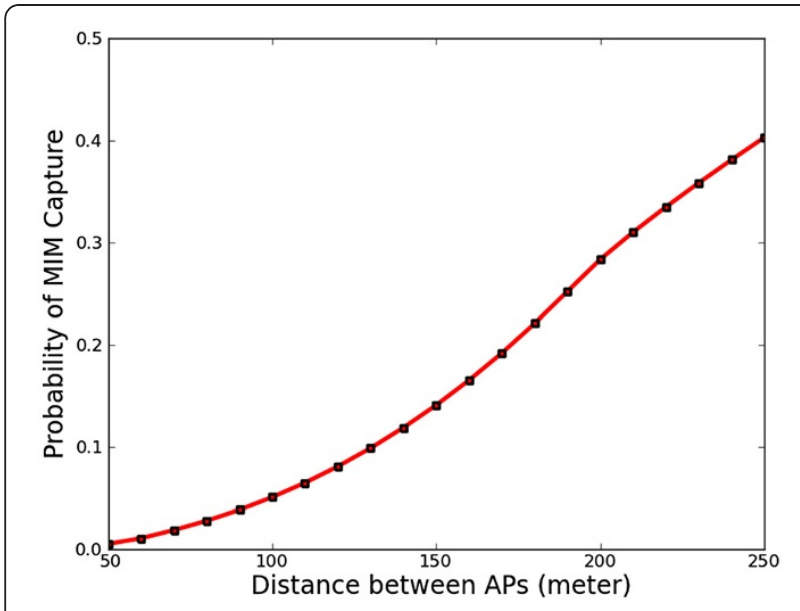

Figure 9 The probability of the MIM capture as a function of distance between the APs.

\section{Simulation results}

\section{Simulation setup}

We implemented the MIM functions of DOMCT in the ns-2 simulator [38] to compare with the legacy DCF and Shuffle. Note that most of the core functions of Shuffle (e.g., packet reordering) have been implemented. However, for fair comparison, we did not use the block ACK option, since it causes Shuffle-enabled devices to dominate the wireless medium by using consecutive multiple transmissions.

We conduct simulations on the IEEE 802.11a WLANs environment and apply the two-ray ground propagation model. Each AP sends CBR traffic over UDP with 1500bytes frame length. The APs and clients are located in a $1000 \times 1000 \mathrm{~m}^{2}$ network and the simulation time is 50 s. Each AP always has packets on its queue (i.e., saturated). The transmission rate is set to $6 \mathrm{Mbps}$. All nodes are using the same channel since we assume the densely deployed WLANs. Figure 10 illustrates the basic topologies used in the simulation scenario. The aforementioned simulation settings are commonly applied in all scenarios unless otherwise stated.

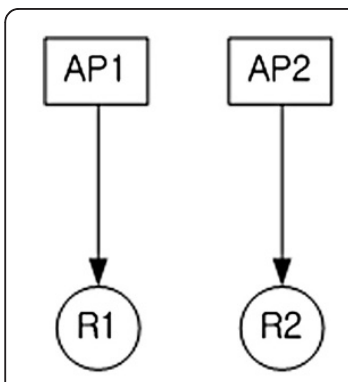

(a)
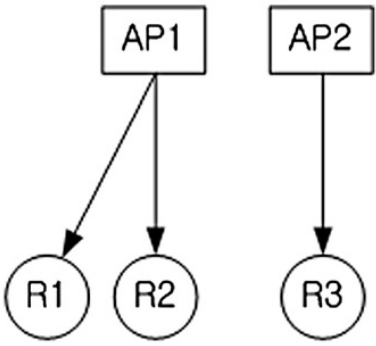

(b)
Figure 10 Simple topologies used in the simulations.

\section{A simple two flows scenario}

Figure 11 shows the MAC layer throughput improvement of DOMCT over DCF as a function of the distance between the two APs as shown in Figure 10a. Note that each AP-STA pair moves together in the same direction in parallel (i.e., the $x$ coordinates of both AP and STA are the same). The throughput improvement represents the ratio between the throughput of DOMCT and that of DCF $\left(\leftrightarrow \frac{\text { throughput of } D O M C T}{\text { throughput of } D C F} \times 100 \%\right)$. The distance between the AP and the client is set to $5 \mathrm{~m}$. DOMCT increases the throughput by up to $61 \%$ if the distance between the APs is greater than $50 \mathrm{~m}$. The results show that the MIM opportunities are increased when the distance from an AP to a client is relatively short and that between the APs is large. The long distance between APs reduces interference and the short distance between an AP and a client increases the SINR. As the distance between the APs increases greater than $200 \mathrm{~m}$, the throughput decreases as the lower SINR is not enough to identify the opportunistic links. In theory, DOMCT may double the throughput of DCF if it has MIM opportunity on every link. Therefore, DOMCT shows better performance than DCF in most cases.

\section{DOMCT with per-station queue}

Figure 12 shows the throughput of DCF, DOMCT, DOMCT with per-station queue (DOMCT + Q), and Shuffle for the topology in Figure 10b. In this article, the throughput is normalized by the throughput gain over DCF unless otherwise stated. DOMCT exploits the opportunistic concurrent transmission technique, while DOMCT + Q adds the per-station queue functionality to DOMCT. In $\mathrm{DOMCT}+\mathrm{Q}$, if an AP has at least one frame for each client and concurrent transmissions are feasible, then the concurrent transmission can be enabled by fetching an

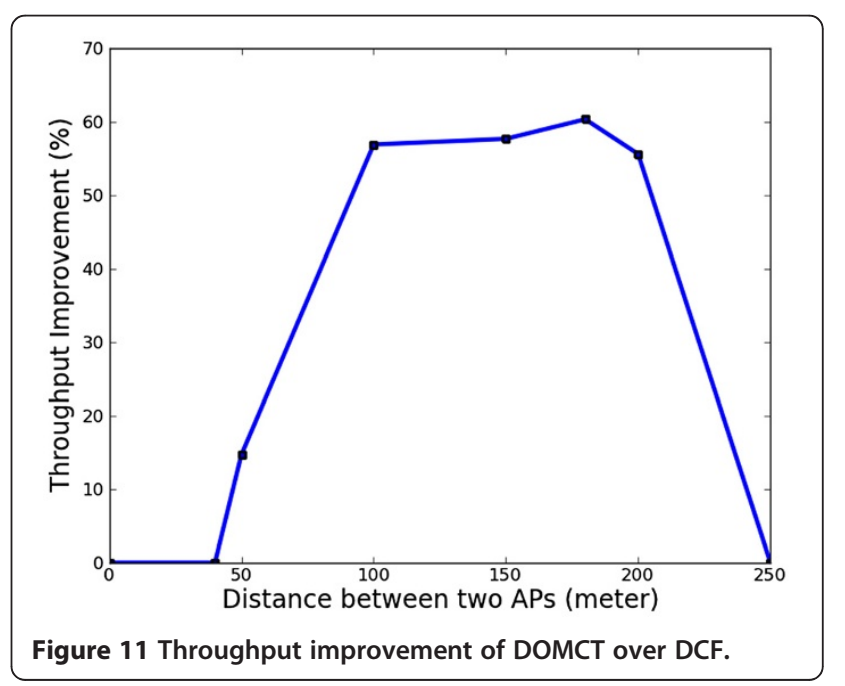




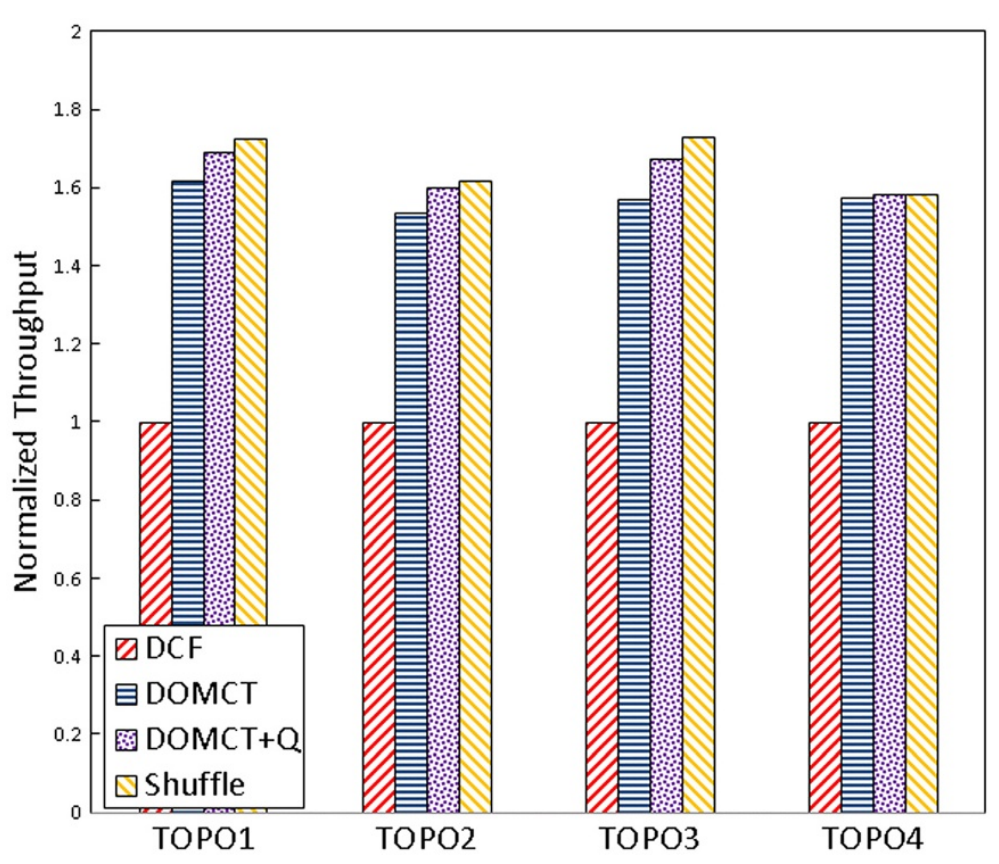

Figure 12 The effect of per-station queue strategy.

appropriate frame from the per-station queue in real-time. We randomly varied the positions of APs and clients in Figure 10b where the association of each AP-STA pair is sustained, and made four different topologies. In these topologies, when AP1 transmits a frame to R2, AP2 has an opportunity to concurrently transmit to R3 in most cases. DOMCT + Q increases the system throughput by up to $10 \%$ compared to DOMCT. The probability of occurring $\mathrm{DOMCT}+\mathrm{Q}$ is expressed as the multiplication between the following two probabilities: (i) the probability that the ongoing transmission of the other AP satisfies MIM constraints on more than one link of an opportunistic AP, and (ii) the probability that the frame on the FIFO queue does not have any concurrent transmission opportunity. Hence, the performance of DOMCT + Q may vary significantly depending on the topology. Shuffle shows better throughput performance than that of DOMCT + Q; thanks to the centralized scheduling that exploits the entire network information. Yet, the difference is not much since DOMCT $+Q$ exploits almost all possible concurrent transmissions.

\section{DOMCT with TR}

To see the effect of the TR, we conduct simulations with VoD applications. The inter packet generation time of each VoD session is set to 1200 pkts/s. We varied the number of $\mathrm{VoD}$ sessions from 2 to 5 and each AP-STA pair (i.e., one VoD session) is randomly located in a $1 \times 1 \mathrm{~km}^{2}$ area. All other settings are the same as described in "Simulation setup" section.
Figure 13 shows the aggregated throughput of the DCF, DOMCT, DOMCT with TR (DOMCT + TR), and Shuffle. DOMCT, DOMCT + TR, and Shuffle outperform the DCF regardless of the number of $\mathrm{VoD}$ sessions due to the power of concurrent transmissions. We observe that the performance gain of DOMCT over DCF is irregular while DOMCT + TR shows stable throughput improvement over DCF. DOMCT try to increase the concurrency of its own transmission, while DOMCT + TR enhances its own concurrency as well as the concurrency of the neighbors. As stated in "DOMCT protocol" section, DOMCT + TR can be activated where the traffic with persistent sending rate such as VoIP and VoD since it needs to know the

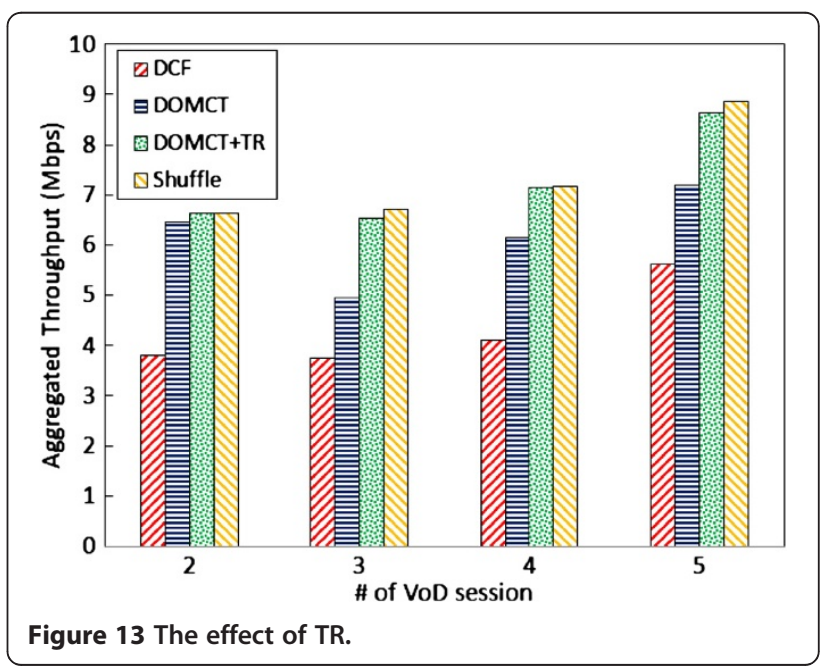


transmission interval of the other APs to regulate the transmission order. Shuffle finds out the optimal set of concurrent transmission links via packet reordering and this advanced operation is very similar to that of DOMCT + TR, thereby both schemes show close throughput performance in these traffic environments.

\section{Broadcast and unicast under a mobility scenario}

In this section, we study the impact of mobility on DCF, DOMCT, and Shuffle. We further categorize DOMCT into unicast (DOMCT-UC) and broadcast transmissions (DOMCT-BC) in order to study the effect of the ACK packets. We disabled the block ACK option and applied a unicast operation to Shuffle as described in "Simulation setup" section. In these experiments, we used the topology shown in Figure 10a. In the mobile scenario, the APs are fixed while the clients move randomly following the random waypoint mobility model with a random speed, random pause time, and to a random destination.

Figure 14 shows the normalized throughput of DCF, DOMCT-UC, DOMCT-BC, and Shuffle. We run the experiment ten times and averaged the results. From the results, we observe that the mobility does not seriously affect the DCF. The performance of DOMCT-BC is degraded by mobility, showing $3 \%$ throughput reduction. This is mainly due to the invalidity in the IMAP table. In the meantime, mobility decreases the throughput of DOMCT-UC by $7 \%$. Note that DOMCT-UC may cause ACK collisions intermittently while DOMCT-BC is free from ACK collisions. Shuffle in the unicast mode shows very similar performance results to that of DOMCT-UC, as expected. In summary, considering the benefits from concurrent transmissions, we observe that DOMCT maintains its performance in the mobile environment regardless of the usage of ACKs. We give the following three reasons: (i) ACK packets are ordered in sequence

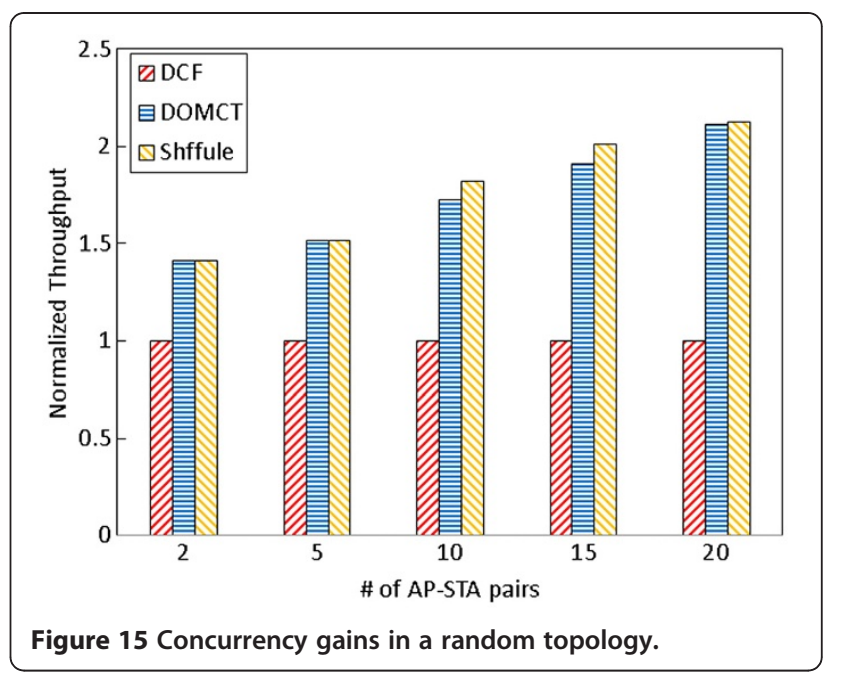

to reduce ACK collisions. (ii) DOMCT uses data packets as well as ACK packets to determine the concurrent transmission success, so that the effect of ACK collisions is minimized. (iii) Each time the AP carries out a concurrent transmission, the result such as success or failure is updated in the IMAP in an opportunistic manner. This keeps the IMAP entries up-to-date so that it can cope with the time-varying channel and user mobility.

\section{Performances in a random topology}

Until now, we have performed the simulations in smallscale environments. Next, we conduct throughput comparison in a larger random topology.

We varied the number of AP-station pairs from 2 to 25 and each AP-STA pair is randomly located in a $1 \times 1$ $\mathrm{km}^{2}$. Only the downlink traffic is generated at each AP. We conducted ten runs and the results were averaged.

Figure 15 shows the normalized throughput of DCF, DOMCT, and Shuffle as a function of the number of

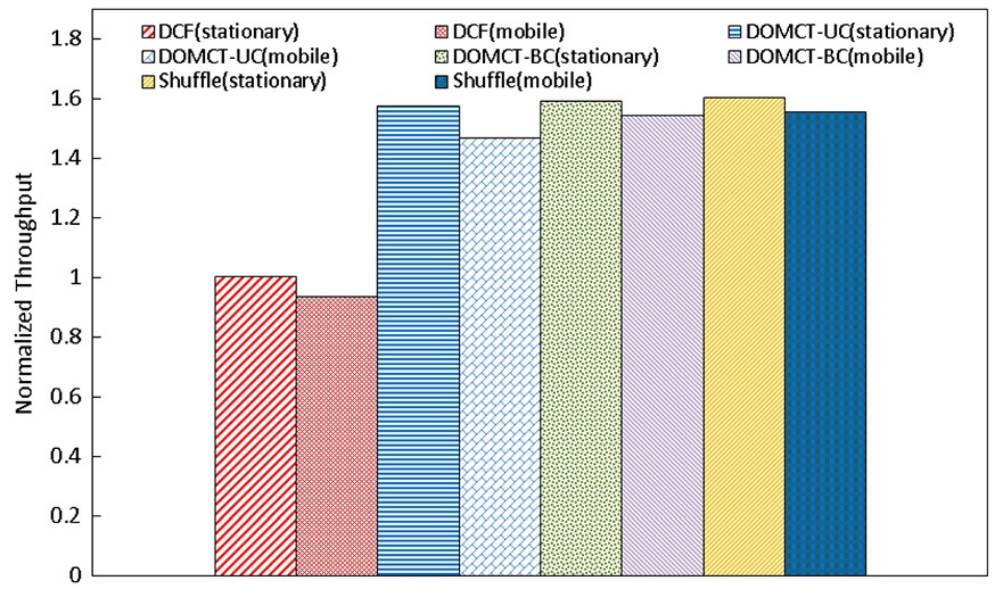

Figure 14 Unicast versus broadcast in a mobile scenario. 
AP-STA pairs. As the number of AP-STA pair increases, the throughput gain of Shuffle and DOMCT over DCF also increases. The reason is that, while DCF experiences more contentions between APs, DOMCT and Shuffle get more concurrent transmission opportunities. On the other hand, Shuffle consistently outperforms the other two methods since it exploits more information such as scheduling information of all APs in the entire network. Nevertheless, DOMCT offers close throughput compared to Shuffle, as it takes advantage of almost every possible concurrent transmission opportunities. As a matter of fact, the throughput performance of the centralized approaches can be viewed as the upper-bound for other distributed methods. However, the centralized approach can be deployed only in a single administrative WLANs environment. Furthermore, it needs to disable the carrier sense mechanism to increase the concurrency, so that other co-existing legacy devices that use DCF will starve due to the unfairness. In contrast, DOMCT is a distributed solution and thereby has the flexibility to be either employed as a standalone solution or adopted partially into the legacy DCF based systems.

\section{Conclusions}

In this article, we proposed an DOMCT protocol for IEEE 802.11 WLANs to improve the system throughput. DOMCT increases the concurrency by opportunistically transmitting a frame immediately after the MAC header of an ongoing frame if the MIM capture threshold requirements are satisfied. As shown in our ns-2 simulations, DOMCT increases the system throughput by up to $61 \%$ higher compared to DCF and achieves close throughput performance compared to Shuffle, the state-of-the-art centralized solution. We are planning to implement DOMCT in a real testbed to verify the feasibility of DOMCT in practice. Also, we will extend DOMCT to be integrated with ad-hoc and mesh networks. We expect DOMCT will enlarge the concurrent transmission chances in ad-hoc and mesh networks since DOMCT is not limited to the downlink traffic only in these networks.

\section{Competing interests}

The authors declare that they have no competing interests.

\section{Acknowledgment}

This work was supported in part by the National Research Foundation of Korea(NRF) grant funded by the Korean government(MEST)

(No.201208302002) and by the Seoul R\&BD Program (WR080951).

\section{Author details}

'Department of Computer Science and Engineering, Seoul National University, Seoul, Korea. ${ }^{2}$ Department of Software Design and Management, Gachon University, Seongnam, Korea. ${ }^{3}$ Electronics and Telecommunications Research Institute, Daejeon, Korea.

Received: 25 May 2012 Accepted: 20 September 2012

Published: 30 October 2012

\section{References}

1. IEEE 802.11n-2009_Amendment 5: enhancements for higher throughput. IEEESA. 29 October 2009 (IEEESTD, 2009), p. p. 5307322

2. B Alawieh, $Y$ Zhang, C Assi, H Mouftah, Improving spatial reuse in multihop wireless networks—a survey. IEEE Commun. Surv. Tutor. 11, 71-91 (2009)

3. P Gupta, PR Kumar, The capacity of wireless networks. IEEE Trans. Inf. Theory 46(2), 388-404 (2000)

4. M Heusse, F Rousseau, R Guillier, A Duda, Idle sense: an optimal access method for high throughput and fairness in rate diverse wireless LANs. ACM SIGCOMM Comput. Commun. Rev. 35(4), 121-131 (2005)

5. F Calì, M Conti, E Gregori, Dynamic tuning of the IEEE 802.11 protocol to achieve a theoretical throughput limit. IEEE/ACM Trans. Netw. 8(6), 785-799 (2000)

6. X Yang, N Vaidya, On the physical carrier sense in wireless Ad-Hoc networks, in Proceedings of IEEE INFOCOM (, Miami, FL, 2005), pp. pp. 2525-pp. 2535

7. Z Zeng, Y Yang, JC Hou, How physical carrier sense affects system throughput in IEEE 802.11 wireless networks, in Proceedings of IEEE INFOCOM (Phoenix, AZ, 2008), pp. pp. 1445-pp. 1453

8. X Liu, A Sheth, M Kaminsky, K Papagiannaki, S Seshan, P Steenkiste, DIRC: increasing indoor wireless capacity using directional antennas, in Proceedings of ACM SIGCOMM (Barcelona, Spain, 2009), pp. pp. 171-pp. 182

9. A Kochut, A Vasan, AU Shankar, A Agrawala, Sniffing out the correct physical layer capture model in $802.11 \mathrm{~b}$, in Proceedings of ICNP (Berlin, Germany, 2004), pp. pp. 252-pp. 261

10. J Lee, W Kim, S Lee, D Jo, J Ryu, T Kwon, Y Choi, An experimental study on the capture effect in 802.11a networks, in Proceedings of WinTECH (Montréal, Québec, 2007), pp. pp. 19-pp. 26

11. J Lee, J Ryu, S Lee, K Ted Taekyoung, Improved modeling of IEEE 802.11a $\mathrm{PHY}$ through fine-grained measurements. Elsevier Comput. Netw. (ComNet) 54(4), 641-657 (2010)

12. N Santhapuri, J Manweiller, S Sen, RR Choudhury, S Nelakuditi, K Munagala, Message in message (MIM): a case for reordering transmissions in wireless networks, in Proceedings of ACM HotNets VII (Calgary, Alberta, 2008), pp. pp. 25-pp. 30

13. J Boer, HV Bokhorst, A Kamerman, R Mud, HV Driest, RJ Kopmeiners, Wireless LAN with enhanced capture provision, Patent 5987033 (US, 1999)

14. Atheros communication, inc. http://www.atheros.com/

15. J Manweiler, N Santhapuri, S Sen, RR Choudhury, S Nelakuditi, K Nunagala, Order matters: transmission reordering in wireless networks, in Proceedings of Mobicom (Beijing, China, 2009), pp. pp. 61-pp. 72

16. H Chang, V Misra, D Rubenstein, A general model and analysis of physical layer capture in 802.11 networks, in Proc IEEE Infocom (Barcelona, Spain, 2006), pp. pp. 1-pp. 12

17. M Durvy, O Dousse, P Thiran, Modeling the 802.11 protocol under different capture and sensing capabilities, in Proceedings of IEEE Infocom (Alaska, US, 2007), pp. pp. 2356-pp. 2360

18. T Nadeem, L Ji, Location-aware ieee 802.11 for spatial reuse enhancement. IEEE Trans. Mob. Comput. 6(10), 1171-1184 (2007)

19. K Whitehouse, A Woo, F Jiang, J Polarstre, D Culler, Exploiting the capture effect for collision detection and recovery, in Proceedings of Emnets, 2005, pp. $45-$-pp. 52

20. D Halperin, T Anderson, D Wetherall, Taking the sting out of carrier sense: interference cancellation for wireless LANs, in Proceedings of Mobicom (San Francisco, US, 2008), pp. 339-pp. 350

21. S. Gollakota, S.D. Perli, D. Katabi, Interference alignment and cancellation, in Proceedings of SIGCOMM (, Barcelona, Spain, 2009), pp. pp. 159-pp. 170

22. K Tan, H Liu, J Fang, W Wang, M Chen, GM Voelker, SAM: enabling practical spatial multiple access in wireless LAN, in Proceedings of Mobicom (Beijing, China, 2009), pp. 49-pp. 60

23. S Gollakota, D Katabi, Zigzag decoding: combating hidden terminals in wireless networks, in Proceedings of SIGCOMM (Seattle, US, 2008), pp. $159-$-pp. 170

24. K Jamieson, H Balakrishnan, PPR: partial packet recovery for wireless networks, in Proceedings of SIGCOMM (Kyoto, Japan, 2007), pp. 409-pp. 420

25. S Sen, R Roy Choudhury, S Nelakuditi, CSMA/CN: carrier sense multiple access with collision notification, in Proceedings of Mobicom (Chicago, USA, 2010), pp. 25-pp. 36

26. V Shrivastava, N Ahmed, S Rayanchu, S Banerjee, S Keshav, K Papagiannaki, A Mishra, CENTAUR: realizing the full potential of centralized WLANs through a hybrid data path, in Proceedings of Mobicom (Beijing, China, 2009), pp. 297-pp. 308 
27. N Ahmed, V Shrivastava, A Mishra, S Banerjee, S Keshav, K Papagiannaki, Interference mitigation in enterprise WLANs through speculative scheduling, in Proceeding of Mobicom (New York, NY, 2007), pp. 342-pp. 345

28. R Murty, A Wolman, J Padhye, M Welsh, An architecture for extensible wireless LANs, in Proceedings of ACM HotNetsVII (Calgary, Alberta, 2008), pp. $79-$ pp. 84

29. R Murty, J Padhye, R Chandra, A Wolman, B Zill, Designing high performance enterprise wi-fi networks, in Proceedings of NSDI (San Francisco, CA, 2008), pp. 73-pp. 88

30. R Murty, J Padhye, A Wolman, M Welsh, Dyson: an architecture for extensible wireless lans, in Proceedings of USENIX Annual Technical Conference (San Francisco, 2010), pp. 201-pp. 214

31. M Vutukuru, $\mathrm{K}$ Jamieson, $\mathrm{H}$ Balakrishnan, Harnessing exposed terminals in wireless networks, in Proceedings of NSDI (San Francisco, CA, 2008), pp. 59-pp. 72

32. C Chen, G Liang, N Vaidya, OCP: opportunistic carrier prediction for wireless networks, in Proceedings of MASS (San Francisco, USA, 2010), pp. 1-pp. 10

33. N Ahmed, U Ismail, S Keshav, K Papagiannaki, Online estimation of RF interference, in Proceedings of CoNext (Spain, Madrid, 2008)

34. Y Kang, J Lee, C Kim, An opportunistic MIM-aware concurrent transmission protocol in IEEE902.11 WLANs, in Proceedings of ICOIN (Kuala Lumpur, Malaysia, 2011), poster 1P-poster 17P

35. J Choi, J Na, Y Lim, K Park, C Kim, Collision-aware design of rate adaptation for multi-rate 802.11 WLANs. IEEE J. Sel. Areas Commun. (JSAC) 26(8), 1366-1375 (2008)

36. S Liu, K-W Fan, P Sinha, CMAC: an energy efficient MAC layer protocol using convergent packet forwarding for wireless sensor networks, in Proceedings of SECON (San Diego, CA, 2007), pp. 11-pp. 20

37. J Lee, Y Kang, C Kim, Opportunities of MIM capture in IEEE 802.11 WLANs: analytic study, in Proceedings of ICUIMC (Seoul, Korea, 2011), pp. 66-pp. 66

38. Ns-2 simulator. http://www.isi.edu/nsnam/ns/

doi:10.1186/1687-1499-2012-325

Cite this article as: Kang et al:: A distributed message in message aware concurrent transmission protocol in IEEE 802.11 WLANs. EURASIP

Journal on Wireless Communications and Networking 2012 2012:325.

\section{Submit your manuscript to a SpringerOpen ${ }^{\circ}$ journal and benefit from:}

- Convenient online submission

- Rigorous peer review

- Immediate publication on acceptance

- Open access: articles freely available online

- High visibility within the field

- Retaining the copyright to your article

Submit your next manuscript at $\gg$ springeropen.com 